\title{
ON SOME FURTHER WIRTINGER-BEESACK INTEGRAL INEQUALITIES
}

\section{Introduction}

In the previous paper [5] the uniform method of obtaining and investigating various types of integral inequalities involving a function and its first derivative (see [4],[2], [7] and [3]) has been extended to the integral inequalities involving a function and its second derivative. Some quadratic integral inequalities of the second order of the form

$$
\int_{I} s h^{2} d t \leq \int_{I} r h^{\prime \prime 2} d t, \quad h \in H,
$$

where $I=(\alpha, \beta),-\infty \leq \alpha<\beta \leq \infty, r$ and $s$ are real functions of the variable $t, H$ is a class of functions absolutely continuous on $I$ has been derived.

In this paper we derive some new integral inequalities of the form (1). The method we use consists in determining the function $s$ and the auxiliary functions $w_{0}, w_{1}$ and $w_{2}$ depending on the given function $r$ and the auxiliary function $\varphi$ and next using these functions to determine the class $H$ of functions $h$ for which the inequality (1) holds. The class of functions $h$ for which the inequality (1) holds determined in this paper doesn't cover with the class of functions $h$ obtained in the paper [5].

We also derive some new integral inequalities of the form (1) with the Chebyshev weight functions $r=\left(1-t^{2}\right)^{a}$ and $s=\gamma\left(1-t^{2}\right)^{b}$ on $I=(-1,1)$, where $a, b, \gamma>0$ are certain constants.

The integral inequalities of the form (1) have been also obtained before, but another methods were applied (for references see e.g. [5], [6], [8]). In the particular case when the interval $I$ is compact and the weight function $r=1$ on $I$ these inequalities were thoroughly studied by Beesack in the 
classic paper [1] and therefore we called the inequalities of the form (1) the Wirtinger-Beesack inequalities.

\section{Main results}

Let $I=(\alpha, \beta),-\infty \leq \alpha<\beta \leq \infty$, be an arbitrary open interval. We denote by $A C(I)$ the class of real functions defined and absolutely continuous on the interval $I$, and by $A C^{1}(I)$ the class of functions $f \in A C(I)$ such that $f^{\prime} \in A C(I)$. Let $r \in A C(I)$ and $\varphi \in A C^{1}(I)$ be given functions such that $r>0, \varphi>0$ on the interval $I$ and $r \varphi^{\prime \prime} \in A C^{1}(I)$. Let us put

$$
s=\left(r \varphi^{\prime \prime}\right)^{\prime \prime} \varphi^{-1} .
$$

Let us denote by $H$ the class of functions $h \in A C^{1}(I)$ satisfying the following integral conditions

$$
\int_{I} r h^{\prime 2} d t<\infty, \quad \int_{I} s h^{2} d t>-\infty
$$

and the limit conditions

$$
\begin{gathered}
\liminf \inf _{t \rightarrow \alpha}\left(w_{0} h^{2}+2 w_{1} h h^{\prime}+w_{2}{h^{\prime 2}}^{2}<\infty,\right. \\
\lim \sup _{t \rightarrow \beta}\left(w_{0} h^{2}+2 w_{1} h h^{\prime}+w_{2}{h^{\prime 2}}^{2}>-\infty\right.
\end{gathered}
$$

and

$$
\liminf _{t \rightarrow \alpha}\left(w_{0} h^{2}+2 w_{1} h h^{\prime}+w_{2} h^{\prime 2}\right) \leq \limsup _{t \rightarrow \beta}\left(w_{0} h^{2}+2 w_{1} h h^{\prime}+w_{2} h^{\prime 2}\right),
$$

where

$$
\begin{gathered}
w_{0}=r\left(\varphi^{-1} \varphi^{\prime}\right)^{3}+r \varphi^{\prime \prime}\left(\varphi^{-1}\right)^{\prime}-\left(r \varphi^{\prime \prime}\right)^{\prime} \varphi^{-1}, \\
w_{1}=r\left(\varphi^{-1} \varphi^{\prime}\right)^{\prime}, \\
w_{2}=r \varphi^{-1} \varphi^{\prime} .
\end{gathered}
$$

Assumptions apply that $w_{0} \in A C(I)$ and $w_{1}, w_{2} \in A C^{1}(I)$.

The following theorem is the main result of this paper:

ThEOREM 1. Let

$$
w=\left(r \varphi^{\prime}\right)^{\prime} \varphi+2 r \varphi \varphi^{\prime \prime}-2 r \varphi^{\prime 2} \leq 0
$$

almost everywhere on the interval $I$.

Then for every function $h \in H$ the inequality

$$
\int_{I} s h^{2} d t \leq \int_{I} r h^{\prime \prime 2} d t
$$

holds.

If $w \not \equiv 0$ and $h \not \equiv 0$ then the inequality (10) becomes an equality if and only if $h=c \varphi$ with $c=$ const $\neq 0$ and the additional conditions 
(11)

$$
\begin{gathered}
\varphi \in H \\
\lim _{t \rightarrow \alpha}\left(w_{0} \varphi^{2}+2 w_{1} \varphi \varphi^{\prime}+w_{2} \varphi^{\prime 2}\right)=\lim _{t \rightarrow \beta}\left(w_{0} \varphi^{2}+2 w_{1} \varphi \varphi^{\prime}+w_{2}{\varphi^{\prime}}^{2}\right)
\end{gathered}
$$

are satisfied.

Proof. This proof is a modification of the proof of Theorem 1 in [5]. Let $h \in A C^{1}(I)$. By virtue of (6), (7), (8) and from assumptions we have $w_{0} h^{2}+2 w_{1} h h^{\prime}+w_{2} h^{\prime 2} \in A C(I)$ and $h \varphi^{-1} \in A C^{1}(I)$. It has been shown in [5] that the identity

$$
r h^{\prime 2}-s h^{2}=\left(v_{0} h^{2}+2 v_{1} h h^{\prime}\right)^{\prime}+g,
$$

where $v_{0}=r \varphi^{\prime \prime}\left(\varphi^{-1}\right)^{\prime}-\left(r \varphi^{\prime \prime}\right)^{\prime} \varphi^{-1}, v_{1}=r \varphi^{\prime \prime} \varphi^{-1}$ and

$$
g=r\left[\varphi\left(\varphi^{-1} h\right)^{\prime \prime}+2 \varphi^{\prime}\left(\varphi^{-1} h\right)^{\prime}\right]^{2}-2 r \varphi \varphi^{\prime \prime}\left[\left(\varphi^{-1} h\right)^{\prime}\right]^{2}
$$

is valid almost everywhere on $I$.

Now we transform the right side of the identity (12) and, after simple calculations, obtain the identity

$$
r h^{\prime \prime 2}-s h^{2}=\left(w_{0} h^{2}+2 w_{1} h h^{\prime}+w_{2} h^{2}\right)^{\prime}+g_{1}
$$

where

$$
g_{1}=r\left[\varphi\left(\varphi^{-1} h\right)^{\prime \prime}+\varphi^{\prime}\left(\varphi^{-1} h\right)^{\prime}\right]^{2}-w\left[\left(\varphi^{-1} h\right)^{\prime}\right]^{2}
$$

which is valid a.e. on $I$. We note that according to the assumptions $g_{1} \geq 0$ a.e on $I$.

Now let $h \in H$. The first condition of (3) implies that the function $r h^{\prime \prime 2}$ is summable on $I$ since $r h^{\prime \prime} \geq 0$ on $I$. It follows from assumptions that the functions $s h^{2}$ and $w_{0} h^{2}+2 w_{1} h h^{\prime}+w_{2} h^{\prime 2}$ are summable on each compact interval $[a, b] \subset I$. Thus by (14) we get the summability of the function $g_{1}$ on $[a, b] \subset I$ and we obtain the equality

$$
\int_{a}^{b} r h^{\prime 2} d t=\int_{a}^{b} s h^{2} d t+\left.\left(w_{0} h^{2}+2 w_{1} h h^{\prime}+w_{2} h^{\prime 2}\right)\right|_{a} ^{b}+\int_{a}^{b} g_{1} d t
$$

for arbitrary $\alpha<a<b<\beta$. In view of (4) there exist two sequences $\left\{a_{n}\right\}$ and $\left\{b_{n}\right\}$ such that $\alpha<a_{n}<b_{n}<\beta, a_{n} \rightarrow \alpha, b_{n} \rightarrow \beta$ and

$$
\begin{aligned}
& \left.\lim _{n \rightarrow \infty}\left(w_{0} h^{2}+2 w_{1} h h^{\prime}+w_{2} h^{\prime 2}\right)\right|_{a_{n}}<\infty, \\
& \left.\lim _{n \rightarrow \infty}\left[-\left(w_{0} h^{2}+2 w_{1} h h^{\prime}+w_{2} h^{\prime 2}\right)\right]\right|_{b_{n}}<\infty .
\end{aligned}
$$

Thus there is a constant $C$ such that

$$
-\left.\left(w_{0} h^{2}+2 w_{1} h h^{\prime}+w_{2} h^{\prime 2}\right)\right|_{a_{n}} ^{b_{n}} \leq C<\infty .
$$

By virtue of the condition $g_{1} \geq 0$ a.e. on $I$ and from the equality (16) we 
infer that

$$
\int_{a_{n}}^{b_{n}} s h^{2} d t \leq \int_{a_{n}}^{b_{n}} r h^{\prime \prime 2} d t+C \leq \int_{I} r h^{\prime \prime 2} d t+C
$$

and from this letting $n \rightarrow \infty$ we obtain

$$
\int_{I} s h^{2} d t \leq \int_{I} r h^{\prime \prime 2} d t+C<\infty .
$$

By this estimate and by the second condition of (3) we conclude that $s h^{2}$ is summable on $I$. Next in a similar way using (16) and the summability of the function $s h^{2}$ on $I$ we prove that the function $g_{1}$ is summable on $I$. Thus all the integrals in the equality (16) have finite limits as $a \rightarrow \alpha$ or $b \rightarrow \beta$, and hence both of the limits in (4) are proper and finite. Therefore the conditions (4) and (5) may be written in the equivalent form

$$
\begin{aligned}
-\infty<\lim _{t \rightarrow \alpha}\left(w_{0} h^{2}+2 w_{1} h h^{\prime}\right. & \left.+w_{2} h^{\prime 2}\right) \\
& \leq \lim _{t \rightarrow \beta}\left(w_{0} h^{2}+2 w_{1} h h^{\prime}+w_{2} h^{\prime 2}\right)<\infty
\end{aligned}
$$

Now by (16) as $a \rightarrow \alpha$ and $b \rightarrow \beta$ we obtain the equality

$$
\begin{aligned}
& \int_{I} r h^{\prime \prime 2} d t-\int_{I} s h^{2} d t \\
= & \lim _{t \rightarrow \beta}\left(w_{0} h^{2}+2 w_{1} h h^{\prime}+w_{2} h^{\prime 2}\right)-\lim _{t \rightarrow \alpha}\left(w_{0} h^{2}+2 w_{1} h h^{\prime}+w_{2} h^{\prime 2}\right)+\int_{I} g d t
\end{aligned}
$$

whence, in view of ( $)$, the inequality (10) follows since $g_{1} \geq 0$ a.e. on $I$.

If the inequality (10) becomes an equality for a non-vanishing function $h \in H$, then by () and (18) we have

$$
\begin{aligned}
\lim _{t \rightarrow \alpha}\left(w_{0} h^{2}+2 w_{1} h h^{\prime}+w_{2} h^{\prime 2}\right) & =\lim _{t \rightarrow \beta}\left(w_{0} h^{2}+2 w_{1} h h^{\prime}+w_{2} h^{\prime 2}\right), \\
\int_{I} g_{1} d t & =0 .
\end{aligned}
$$

As $g_{1} \geq 0$ a.e. on $I$ we obtain $g_{1}=0$ a.e. on $I$. Hence

$$
\varphi\left(\varphi^{-1} h\right)^{\prime \prime}+\varphi^{\prime}\left(\varphi^{-1} h\right)^{\prime}=0
$$

a.e. on $I$ and $w\left[\left(\varphi f^{2}\right)^{\prime}\right]^{2}=0$ a.e. on $I$. According to the assumptions $w \not \equiv 0$ and whence there exists $t_{0} \in I$ such that $\left(\varphi^{-1} h\right)^{\prime}\left(t_{0}\right)=0$. The function $\left(\varphi^{-1} h\right)^{\prime} \in A C(I)$ satisfies the homogeneous linear differential equation (20) with the initial value $\left(\varphi^{-1} h\right)^{\prime}\left(t_{0}\right)=0$, and whence $\left(\varphi^{-1} h\right)^{\prime}=0$ on $I$. This implies that $h=c \varphi$, where $c=$ const $\neq 0$, since $\varphi^{-1} h \in A C^{1}(I)$. Thus $\varphi \in H$ so that we obtain the first condition of (11) and from the second condition of (19) we get the second condition of (11). 
Now let (11) be satisfied and let $h=c \varphi$, where $c=$ const $\neq 0$. That implies $g_{1}=0$ a.e. on $I$ so that $\int_{I} g_{1} d t=0$ and, in view of (18), the inequality (10) becomes an equality which completes the proof.

REMARK 1 . In case of $r=1$ on $I$ we have $w=3 \varphi \varphi^{\prime \prime}-2 \varphi^{\prime 2}$. Moreover if $-\infty<\alpha<\beta<\infty, \varphi(\alpha)=\varphi^{\prime}(\alpha)=\varphi(\beta)=\varphi^{\prime}(\beta)=0$ and there exist finite limit values $\varphi^{\prime \prime}(\alpha)$ and $\varphi^{\prime \prime}(\beta)$ and $s>0$ on $I$, then it follows from Lemma 2.3 in [1], that $w \leq 0$ on $I$. Therefore under these assumptions Theorem 1 is valid. So that Theorem 2.2 in [1] is a particular case of Theorem 1.

EXAMPLE. Let us take $I=(-1,1)$ and the functions $r=\left(1-t^{2}\right)^{a}$ and $\varphi=\left(1-t^{2}\right)^{2-a}$ on $I$, where $a$ is an arbitrary constant such that $a<1$.Then we have

$$
s=4(a-2)(2 a-3)\left(1-t^{2}\right)^{a-2}>0
$$

and

$$
w=2(a-2)\left(3-t^{2}\right)\left(1-t^{2}\right)^{2-a}<0
$$

on $I$.

From Theorem 1 we obtain that the inequality holds for every function $h \in H$, where $H$ is the class of functions $h \in A C^{1}((-1,1))$ satisfying the integral condition

$$
\int_{-1}^{1}\left(1-t^{2}\right)^{a} h^{\prime \prime 2} d t<\infty
$$

and the limit condition

$$
-\infty<\lim _{t \rightarrow-1} S\left(t, h, h^{\prime}\right) \leq \lim _{t \rightarrow 1} S\left(t, h, h^{\prime}\right)<\infty
$$

where

$$
\begin{aligned}
S\left(t, h, h^{\prime}\right)= & \omega_{0}(t)\left(1-t^{2}\right)^{a-3} h^{2}+\omega_{1}(t)\left(1-t^{2}\right)^{a-2} h h^{\prime} \\
& ++\omega_{2}(t)\left(1-t^{2}\right)^{a-1} h^{\prime 2}
\end{aligned}
$$

and

$$
\begin{aligned}
& \omega_{0}(t)=4(a-2) t\left[(5-3 a)+(a-1) t^{2}\right], \\
& \omega_{1}(t)=4(a-2)\left(1+t^{2}\right), \\
& \omega_{2}(t)=2(a-2) t,
\end{aligned}
$$

since the second condition of $(3)$ is satisfied trivially.

Now we show that a function $h \in A C^{1}((-1,1))$ satisfying the integral condition (21) and the limit conditions

$$
h(-1)=h^{\prime}(-1)=h(1)=h^{\prime}(1)=0
$$

belongs to the class $H$. 
At first we show that if $h(1)=h^{\prime}(1)=0$ and (21) holds, then the limit condition

$$
\lim _{t \rightarrow 1} S\left(t, h, h^{\prime}\right)=0
$$

is satisfied. Let us consider the left-hand neighbourhood $U$ of the point 1 . Using the Schwarz inequality we obtain the estimate

$$
\begin{aligned}
\left|h^{\prime}(t)\right|^{2} & =\left(\int_{t}^{1} h^{\prime \prime} d \tau\right)^{2} \leq \int_{t}^{1}\left(1-\tau^{2}\right)^{-a} d \tau \int_{t}^{1}\left(1-\tau^{2}\right)^{a} h^{\prime \prime 2} d \tau \\
& \leq A \int_{t}^{1}(1-\tau)^{-a} d \tau \int_{t}^{1}\left(1-\tau^{2}\right)^{a} h^{\prime \prime 2} d \tau \\
& =\frac{A}{1-a}(1-t)^{1-a} \int_{t}^{1}\left(1-\tau^{2}\right)^{a} h^{\prime \prime 2} d \tau
\end{aligned}
$$

for $t \in U$, where $A>0$ is a constant such that $(1+t)^{-a} \leq A$ on $U$. Whence we have

$$
\left|h^{\prime}(t)\right| \leq k(t)(1-t)^{\frac{1-a}{2}}
$$

for $t \in U$, where

$$
k(t)=\left\{\frac{A}{1-a} \int_{t}^{1}\left(1-\tau^{2}\right)^{a} h^{\prime \prime 2} d \tau\right\}^{\frac{1}{2}}>0, \quad t \in U
$$

is a continuous function on $I$ such that $\lim _{t \rightarrow 1} k(t) \equiv k(1)=0$. Next using (25) and the Schwartz inequality again we get the estimate

$$
\begin{aligned}
|h(t)|^{2} & =\left|\int_{t}^{1} h^{\prime} d \tau\right|^{2} \leq\left(\int_{t}^{1}\left|h^{\prime}\right| d \tau\right)^{2} \leq\left(\int_{t}^{1} k(\tau)(1-\tau)^{\frac{1-a}{2}} d \tau\right)^{2} \\
& \leq \int_{t}^{1} k^{2}(\tau) d \tau \int_{t}^{1}(1-\tau)^{1-a} d \tau=\frac{1}{2-a} k^{2}(\theta)(1-t)^{3-a}
\end{aligned}
$$

for $t \in U$, where $t<\theta<1$ and, in the last step, we applied Mean Value Theorem for the integral $\int_{t}^{1} k^{2}(\tau) d \tau$. From the above estimate we get

$$
|h(t)| \leq B k(\theta)(1-t)^{\frac{3-a}{2}}
$$

for $t \in U$, where $B=\frac{1}{\sqrt{2-a}}$ and $\lim _{t \rightarrow 1} k(\theta)=k(1)=0$.

Next based on the estimates (25) and (26) we obtain from (23) that

$$
\begin{aligned}
\left|S\left(t, h, h^{\prime}\right)\right| \leq & B^{2}\left|\omega_{0}(t)\right| k^{2}(\theta)(1+t)^{a-3}+B\left|\omega_{1}(t)\right| k(\theta) k(t)(1+t)^{a-2} \\
& +\left|\omega_{2}(t)\right| k^{2}(t)(1+t)^{a-1} \equiv m(t)
\end{aligned}
$$

for $t \in U$ with $\lim _{t \rightarrow 1} m(t)=0$. Whence it follows that $\lim _{t \rightarrow 1} S\left(t, h, h^{\prime}\right)=0$. 
In analogous way we show that if $h(-1)=h^{\prime}(-1)=0$ and (21) holds, then

$$
\lim _{t \rightarrow-1} S\left(t, h, h^{\prime}\right)=0 \text {. }
$$

Based on the above considerations we obtain that from the conditions (21) and (24) follows the condition (22).

Therefore we get the following:

If $a<1$ and the function $h \in A C^{1}((-1,1))$ satisfies the integral condition

$$
\int_{-1}^{1}\left(1-t^{2}\right)^{a} h^{\prime \prime 2} d t<\infty
$$

and the limit conditions

$$
h(-1)=h^{\prime}(-1)=h(1)=h^{\prime}(1)=0
$$

then the inequality

$$
4(a-2)(2 a-3) \int_{-1}^{1}\left(1-t^{2}\right)^{a-2} h^{2} d t \leq \int_{-1}^{1}\left(1-t^{2}\right)^{a} h^{\prime \prime 2} d t
$$

holds.

The inequality (27) becomes an equality if and only if $h=c\left(1-t^{2}\right)^{2-a}$, where $c=$ const.

In the particular case for $a=0$ we obtain the inequality otherwise deduced in [1].

REMARK 2. In the paper [5] the inequality (10) has been derived under the assumption $\varphi^{\prime \prime} \leq 0$ on $I$. In this paper the assumption has been put on both functions $r$ and $\varphi$, and this involves also the cases in which the condition $\varphi^{\prime \prime} \leq 0$ on $I$ is not satisfied. Indeed, the inequality (27) with $a<1$ cannot be obtained from Theorem 1 in [5], as for $a<1$ the function $\varphi=\left(1-t^{2}\right)^{2-a}$ doesn't satisfy the condition $\varphi^{\prime \prime} \leq 0$ on $(-1,1)$. Therefore the classes of functions $h$ for which the inequality (1) holds obtained in [5] and in this paper are not equal.

\section{References}

[1] P. R. Beesack, Integral inequalities of the Wirtinger type, Duke Math. J. 25 (1958), 477-498.

[2] B. Florkiewicz, Some integral inequalities of Hardy type, Colloq. Math. 43 (1980), 321-330.

[3] B. Florkiewicz and M. Kuchta, Some quadratic integral inequalities of first order, Colloq. Math. 75 (1998), 7-18. 
[4] B. Florkiewicz and A. Rybarski, Some integral inequalities of Sturm-Liouville type, Colloq. Math. 36 (1976), 127-141.

[5] B. Florkiewicz and K. Wojteczek, Some second order integral inequalities of generalized Hardy-type, Proc. Royal Soc. Edinburgh A, to appear.

[6] G. H. Hardy, J. E. Littlewood and G. Polya, Inequalities, 2nd ed., Cambridge Univ. Press, Cambridge, 1991.

[7] M. Kuchta, Some quadratic integral inequalities of Opial type, Ann. Polon. Math. 63 (1996), 103-113.

[8] D. S. Mitrinović, J. E. Petarić, and A. M. Fink, Inequalities Involving Functions and Their Integrals and Derivatives, Kluwer Acad. Publ., Dordrecht, 1991.

\author{
Bronisław Florkiewicz \\ INSTITUTE OF MATHEMATICS \\ WROCEAW UNIVERSITY OF TECHNOLOGY \\ Wybrzeże Wyspiańskiego 27 \\ 50-370 WROCEAW, POLAND \\ Katarzyna Wojteczek \\ INSTITUTE OF MATHEMATICS \\ TECHNICAL UNIVERSITY OF OPOLE \\ Luboszycka 3 \\ 45-036 OPOLE, POLAND
}

Received March 11, 1998. 